\title{
Autonomic adaptation after traditional and reverse swimming training periodizations
}

\author{
VJ Clemente-Suárez ${ }^{1,4}$, RJ Fernandes ${ }^{2,3}$, JJ Arroyo-Toledo ${ }^{1}$, P Figueiredo $^{2}$, \\ JM González-Ravé ${ }^{,}$JP Vilas-Boas ${ }^{2,3}$ \\ ${ }^{1}$ Department of Physical Activity and Sport Science. Faculty of Sport Science, University of Castilla la Mancha, \\ Toledo, Spain \\ ${ }^{2}$ Centre of Research, Education, Innovation and Intervention in Sport (CIFI2D), Faculty of Sport, \\ University of Porto, Porto, Portugal \\ ${ }^{3}$ Porto Biomechanics Laboratory (LABIOMEP), University of Porto, Porto, Portugal \\ ${ }^{4}$ Department of Motricity, Human Performance and Sport Management, European University of Madrid, \\ Madrid, Spain
}

Received: October 24, 2013

Accepted after revision: August 21, 2014

\begin{abstract}
The objective of the present study was to analyze the autonomic response of trained swimmers to traditional and reverse training periodization models. Seventeen swimmers were divided in two groups, performing a traditional periodization (TPG) or a reverse periodization (RPG) during a period of 10 weeks. Heart rate variability and $50 \mathrm{~m}$ swimming performance were analyzed before and after the training programs. After training, the TPG decreased the values of the high frequency band (HF), the number of differences between adjacent normal R-R intervals longer than $50 \mathrm{~ms}$ (NN50) and the percentage of differences between adjacent normal R-R intervals more than $50 \mathrm{~ms}$ (pNN50), and the RPG increased the values of HF and square root of the mean of the sum of the squared differences between adjacent normal R-R intervals (RMSSD). None of the groups improved significantly their performance in the 50-m test. The autonomic response of swimmers was different depending on the periodization performed, with the reverse periodization model leading to higher autonomic adaption. Complementary, the data suggests that autonomic adaptations were not critical for the 50-m swimming performance.
\end{abstract}

Keywords: heart rate variability, performance, sympathetic nervous system, parasympathetic nervous system, swimming

Researches in training periodization have frequently been focused on strength and endurance training (18). Variations in volume, intensity and frequency of training have been analyzed in different sports, such as running and swimming $(1,21)$, and in specific periods of periodization as tapering (14). Presently, many sports use traditional training periodization, this model is based on performing high volume and low intensity during the preparatory period and after that phase, volume is slightly reduced and intensity is increased. Conversely, an eventual drop of volume and an increase of intensity is suggested, in order to obtain higher performance and avoid overtraining $(2,26)$. Its efficacy in swimming was already confirmed by Costill et al. (2), but this training paradigm cannot be considered a real periodization model. Meanwhile, different periodization models, such as the block training system (26) and the block

Corresponding author: Vicente Javier Clemente-Suárez

Department of Motricity, Human Performance and Sport Management, European University of Madrid

C/ Tajo s/n, Villaviciosa de Odón, 28670 Madrid, Spain

E-mail: vctxente@yahoo.es 
periodization (11), were proposed with a different paradigm: the specific training loads were concentrated in a sheeted period, but the same distribution from volume to intensity was observed.

Presently a new periodization model is emerging in opposition to the traditional periodization model: the reverse periodization. This new model is also based on the concept of low volume and high intensity $(2,26)$, but is characterized by a completely opposite paradigm regarding the traditional periodization program: the training program begins with high intensity and low volume and in the following periods, the intensity decreases and the volume increases or, in other cases, the intensity is maintained and volume increases, depending on the sport $(4,17,18)$. Reverse periodization was studied in physical fitness, strength training and rowing, obtaining increases in muscular endurance (4), maximum strength (17) and endurance performance (18). In the same line, Gibala et al. (6) have demonstrated that short periods of high intensity training, with adequate rest, produce similar adaptations, as high volume of traditional endurance training, and both endurance and sprintinterval training induced similar increases in muscle buffering capacity and glycogen content.

The control of training workloads has been evaluated by various methods, mainly by the assessment of volumes and intensities (15) and the monitoring of the heart rate during periods of rest (27). Currently, the use of the heart rate variability (HRV) is also used as a method of training control and overtraining states diagnosis (27) and it is considered as an important parameter to assess the adaptive or non-adaptive response to sports training loads (24). Complementary, it has been demonstrated that exercise training results in significant increases in R-R interval (time between two $\mathrm{R}$ waves of the recorded cardiac electrical activity) and high frequency power of HRV (19). Yamamoto et al. (28) have suggested that the increase in HRV induced by exercise training might be caused by a decrease of sympathetic tonus and/ or an increase in vagal tonus. Therefore, the autonomic response depends on the type of exercise performed.

Actually the effect of different periodization models in the organic response of athletes are poorly studied and only focus on traditional physiological parameters $(1,14,18,21)$, autonomic response to training periodization could be an important factor to improve the training loads distribution and improve the adaptative response of athletes. For this reason, the purpose of the present study was to analyze the autonomic response and $50 \mathrm{~m}$ swimming performance to traditional and reverse periodization training models. It was hypothesized that the reverse periodization training model leads to a higher autonomic adaptation and $50-\mathrm{m}$ swimming performance comparing to the traditional periodization training model.

\section{Methods}

\section{Research design}

A pre-post training intervention design was employed with two experimental groups. The investigation aimed to study the changes in autonomic response and 50-m swimming performance after the completion of 10 weeks of traditional and reverse training periodizations in two groups of trained swimmers. Our independent variable was the 10 weeks of traditional and reverse training periodization models and the dependent variables were the HRV and the time in the 50-m swimming test analyzed before and after the 10 weeks of training programs.

\section{Subjects}

Seventeen volunteer swimmers were divided into two groups: traditional periodization group (TPG) ( $n=7$; 4 females: weight $62.5 \pm 6.8 \mathrm{~kg}$; height $1.76 \pm 0.06 \mathrm{~m}$; BMI $20.2 \pm 0.9 \mathrm{~m} / \mathrm{kg}^{2}$; 
age $18.5 \pm 1.9$ years; 3 males: weight $71.0 \pm 3.5 \mathrm{~kg}$; height $1.79 \pm 0.01 \mathrm{~m}$; BMI $22.1 \pm 1.0 \mathrm{~m} /$ $\mathrm{kg}^{2}$; age $17.3 \pm 0.6$ years $)$ and reverse periodization group (RPG) $(n=10 ; 5$ females: weight $54.2 \pm 4.2 \mathrm{~kg}$; height $1.66 \pm 0.08 \mathrm{~m}$; BMI $19.7 \pm 2.1 \mathrm{~m} / \mathrm{kg}^{2}$; age $15.8 \pm 2.6$ years; $5 \mathrm{mal}$; body mass $76.0 \pm 6.2 \mathrm{~kg}$; height $1.79 \pm 0.08 \mathrm{~m}$; BMI $23.9 \pm 2.8 \mathrm{~m} / \mathrm{kg}^{2}$; age $19.3 \pm 3.0$ years). Swimmers came from three different clubs, swimmers of one club performed the traditional periodization and swimmers of the other two clubs performed the reverse periodization. We select this methodology because it was the best option not to interfere with the diary training session since every swimmers of the club performed the same training sessions. Swimmers had $6.5 \pm 4.9$ years of training experience and all of them competed at the national level at the time of the experiments. Prior to participation, the experimental procedures were explained to all participants, who gave their voluntary written informed consent. The study was conducted in accordance with the Declaration of Helsinki.

\section{Procedure}

Both groups of swimmers completed a program of 10 weeks of training: one group performed a traditional periodization model (TPG) and the other one performed a reverse periodization model (RPG). The week before and after the completion of the 10-week training period, each swimmer performed a standardized warm-up consisting of $1500 \mathrm{~m}$ of aerobic swimming, and a 50-m maximum swimming test in a $25 \mathrm{~m}$ indoor swimming pool. The temperature of the air in the facility containing the swimming pool varied between $29-30^{\circ} \mathrm{C}$, the humidity between $47-50 \%$ and the temperature of the water between $27.0-27.5^{\circ} \mathrm{C}$. Before the warmup, swimmers performed a HRV test, which lasted for $10 \mathrm{~min}$ in a supine position, lying in a stretcher in a room with controlled temperature. Pre- and post-training HRV test was conducted at the same time of the day. The R-R interval was measured with a Polar S810 heart rate monitor (Polar, Kempele, Finland). The R-R series were analyzed using Kubios HRV software (version 2.0, Biosignal Analysis and Medical Imaging Group, University of Kuopio, Finland) that was developed in accordance with the literature recommendations (23). This software shows excellent validity and is able to account for non-linear trends often present in beat-to-beat recordings by detrending the filtered R-R data using the smoothness priors approach (7). Data was interpolated at a rate of $4 \mathrm{~Hz}$ in accordance with the software's recommendations (8).

The following HRV variables were assessed: (i) total power; (ii) very low frequency (VLF) band; (iii) low-frequency (LF) band; (iv) high-frequency (HF) band; (v) normalized LF and HF; (vi) LF/HF ratio; (vii) percentage of differences between adjacent normal R-R intervals more than $50 \mathrm{~ms}$ (PNN50); (viii) number of differences between adjacent normal R-R intervals higher than $50 \mathrm{~ms}$ (NN50); (ix) standard deviation of all normal R-R intervals (SDNN); (x) square root of the mean of the sum of the squared differences between adjacent normal R-R intervals (RMSSD); (xi) mean heart rate; and (xii) mean R-R.

\section{Training protocols}

Three training zones were used to control and quantify the volume and intensity of training (22): zone 1, low intensity training ( $<3 \mathrm{mmol} / \mathrm{l}$ of blood lactate concentration); zone 2, anaerobic threshold training (3-4 mmol/l of blood lactate concentration); zone 3 , high intensity training ( $>4 \mathrm{mmol} / \mathrm{l}$ of blood lactate concentration). The TPG began the training period with high volumes in $\mathrm{Z1}$ and $\mathrm{Z2}$, and then, gradually introduced training sessions in Z3. In contrast, the RPG performed trainings in Z3 during the 10 weeks, increasing training volume in $\mathrm{Z} 2$ in the middle weeks of the training period. Whereas the TPG made a progression 
from high volume of training in $\mathrm{Z1}$ and $\mathrm{Z} 2$, to more intense training sessions (Z3) in subsequent weeks, the RPG started with high intensity training that was maintained during the 10 weeks of training.

TPG performed during the 10-week training $293666 \mathrm{~m}$ in zone $1,8300 \mathrm{~m}$ in zone 2, and $35085 \mathrm{~m}$ in zone 3, achieving a total volume of $337051 \mathrm{~m}$. RPG performed $133600 \mathrm{~m}$ in Z1, $12500 \mathrm{~m}$ in Z2, and $12924 \mathrm{~m}$ in Z3, with a total volume of $159024 \mathrm{~m}$. Weekly distributions of training load are showed in Fig. 1. The 10-week program was divided in four phases according to previous literature $(11,26)$, the characteristics and duration of the training exercise in each phase are showed in Table I.
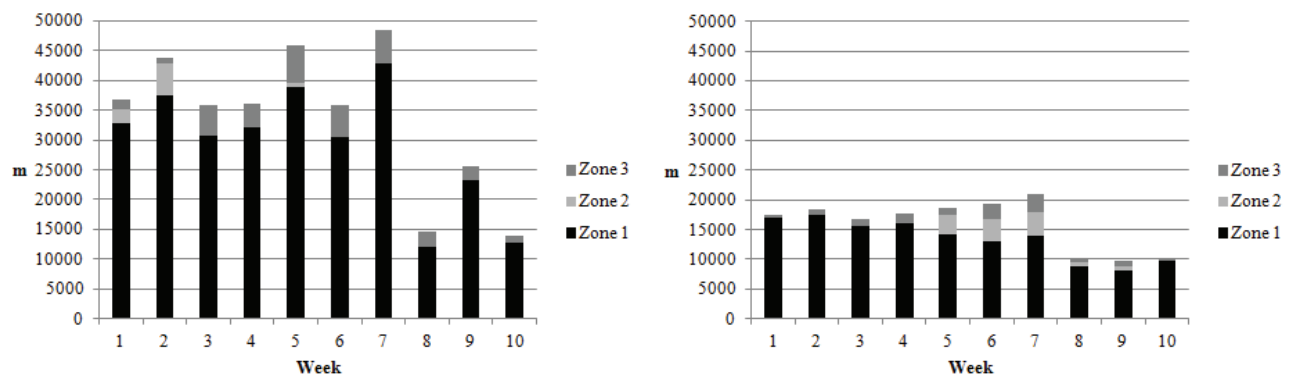

Fig. 1. Distribution of training intensity zones for traditional and the reverse periodization groups (left and right panels, respectively) during the 10 weeks of training

Zone 1: low intensity training $<2 \mathrm{mmol} / \mathrm{l}$. zone 2 : threshold training $3-4 \mathrm{mmol} / \mathrm{l}$ and zone 3 : high intensity training $>4 \mathrm{mmol} / \mathrm{l}$

\section{Statistical analyses}

Data were analyzed using the Statistical Package for the Social Sciences (SPSS) version 17 (SPSS Inc., Chicago, Ill., USA). The Shapiro-Wilk normality test was used to test the normality and homogeneity of each variable. A multiple $t$-test was performed for parametric data (HF, normalized LF and LF, SDNN, mean HR, RMSSD, NN50, pNN50), and a Wilcoxon test for non-parametric data (total power, VLF, LF). The level of significance for all the comparisons was set at $p<0.05$ and the effect size of results was calculated with the Cohen's $D$.

Table I. Examples of typical training series in each phase of the 10-week training period for both traditional and reverse training periodizations

\begin{tabular}{|c|c|c|c|c|}
\hline \multirow[b]{2}{*}{$\begin{array}{l}\text { Periodization } \\
\text { model }\end{array}$} & \multicolumn{4}{|c|}{ Phase } \\
\hline & $\begin{array}{c}\text { I } \\
\text { Weeks 1-3 }\end{array}$ & $\begin{array}{c}\text { II } \\
\text { Weeks 4-6 }\end{array}$ & $\begin{array}{c}\text { III } \\
\text { Weeks 7-8 }\end{array}$ & $\begin{array}{c}\text { IV } \\
\text { Weeks 9-10 }\end{array}$ \\
\hline Tradi & $\begin{array}{l}20 \times 200 \mathrm{~m} \mathrm{Z1} / 10 " \\
5 \times 400 \mathrm{~m} \mathrm{Z1/30"}\end{array}$ & $\begin{array}{l}3 \times(6 \times 100 \mathrm{~m} \mathrm{Z3/30"}) / 3 \\
20 \times 150 \mathrm{~m} \mathrm{Z} 2 / 10 "\end{array}$ & $\begin{array}{l}6 \times\left(3 \times 50 \mathrm{~m} \mathrm{Z3} / 10^{\prime \prime}\right) / 5^{\prime} \\
6 \times 100 \mathrm{~m} \mathrm{Z3/2},\end{array}$ & $\begin{array}{l}8 \times\left(2 \times 25 \mathrm{~m} \mathrm{Z3} / 5^{\prime \prime}\right) 3^{\prime} \\
3 \times 100 \mathrm{~m} \mathrm{Z3/10"}\end{array}$ \\
\hline Reverse & $\begin{array}{l}6 \times 12 \mathrm{~m} \\
\text { with shorts } \mathrm{Z3} / 3 \text {, } \\
10 \times 25 \mathrm{~m} \mathrm{Z3} / 3 \text {, }\end{array}$ & $\begin{array}{l}8 \times 12 \mathrm{~m} \text { with shorts } \mathrm{Z3} / 3^{\prime} \\
4 \times\left(10 \times 15 \mathrm{~m} \mathrm{Z} 3 / 30^{\prime \prime}\right) 3^{\prime} \\
10 \times 200 \mathrm{~m} \mathrm{Z} 2 / 20^{\prime \prime}\end{array}$ & $\begin{array}{l}8 \times 12 \mathrm{~m} \text { with shorts } \mathrm{Z} 3 / 3^{\prime} \\
3 \times\left(16 \times 25 \mathrm{~m} \mathrm{Z3} / 30^{\prime \prime}\right) / 3 \\
4 \times\left(10 \times 15 \mathrm{~m} \mathrm{Z} 3 / 30^{\prime \prime}\right) / 3\end{array}$ & $\begin{array}{l}6 \times 12 \mathrm{~m} \\
\quad \text { with shorts } \mathrm{Z3} / 3, \\
2 \times\left(4 \times 25 \mathrm{~m} \mathrm{Z3} / 30^{\prime \prime}\right) / 8\end{array}$ \\
\hline
\end{tabular}

Series $\times$ (repetition $\times$ distance and intensity / recovery between repetitions) / recovery between series; $Z 1-$ low intensity training; Z2 - threshold training; Z3 - high intensity training; Shorts - weathered swim with short pants 


\section{Results}

The TPG decreased the values of HF, NN50 and pNN50 significantly, after the 10-week training period, and the RPG increased the values of HF and RMMSD significantly (Tables II and III). The other HRV parameters analyzed in both groups did not change significantly with training. The time in the 50 -m test did not decrease significantly $0.09 \%$ in the TPG ( $28.81 \pm 1.72$ vs. $28.78 \pm 1.44 \mathrm{~s}$; Cohen' $D: 0.02)$ and it did not increase significantly $2.3 \%$ in the RPG ( $29.50 \pm 2.07$ vs. $30.24 \pm 2.83 \mathrm{~s}$; Cohen' $D:-0.36)$.
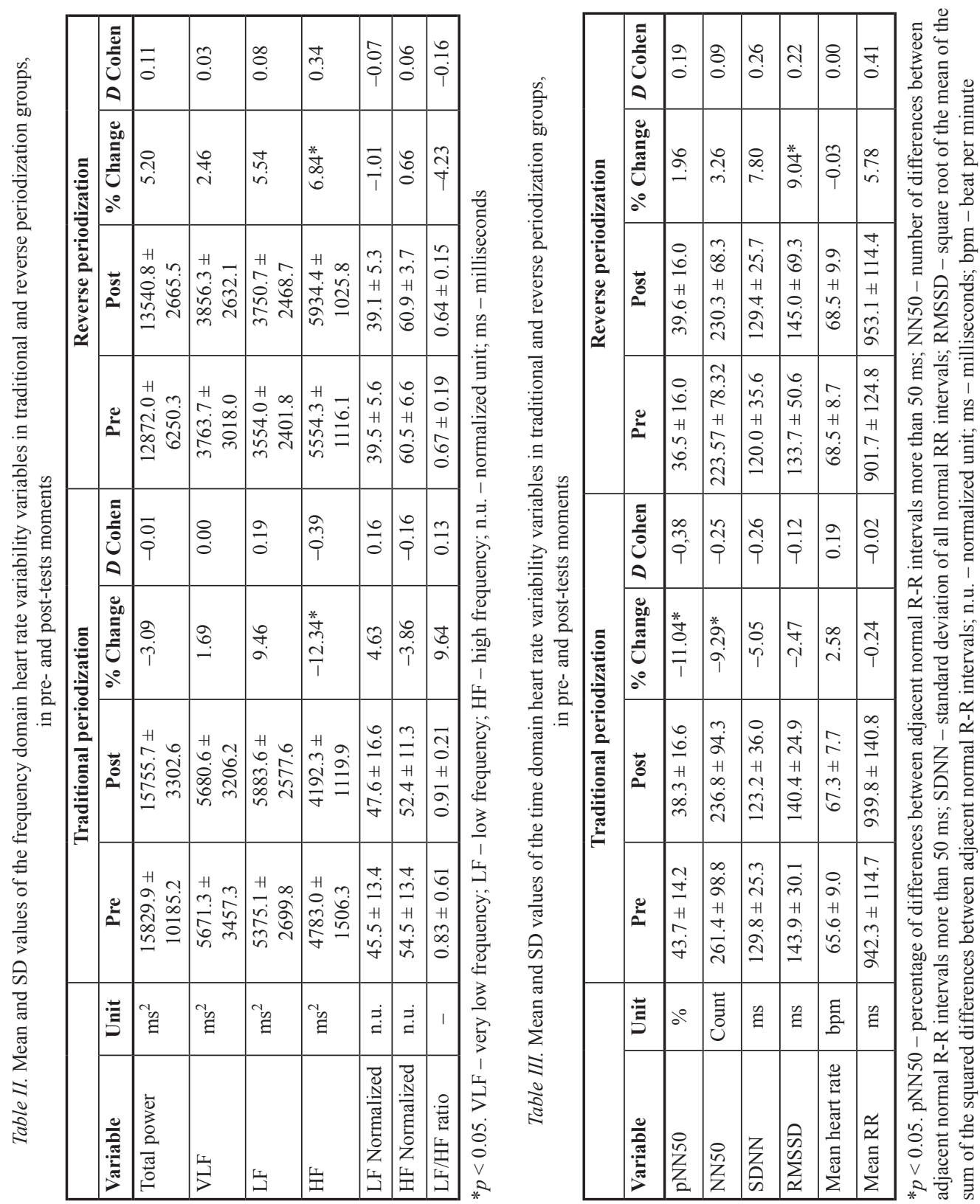


\section{Discussion}

The results obtained in the present study evidenced changes on autonomic response depending on the periodization training model performed (traditional vs. reverse). This is novel and should be taken into account by researchers and coaches. The initial hypothesis was partially complied once the RPG achieved a higher autonomic adaptation than the TPG; however the 50-m performance was not significantly affected by training in either group.

TPG obtained a decrease in HF, in contrast the RPG increased this parameter, reflecting a higher parasympathetic domain in the RPG. By contrast, sympathetic modulation on the TPG was higher comparing to the RPG due to a greater increase in LF (8). The LF increase in the TPG is in accordance with previous studies that found higher values of this parameter in endurance athletes after performing an experimental high volume training program during 6-9 weeks (25). The increase in sympathetic modulation in the TPG is contrary to the lower LF and less training volumes in the RPG, this phenomenon, jointly with the decrease in autonomic adaptation of the TPG have been described in the literature for periods of high training load (10). Conversely, the decrease in sympathetic activity of the RPG was observed after periods of low training load or pre-competitive training periods (10). Moreover, the increase in parasympathetic modulation on the RPG was confirmed by the increase in RMSSD, NN50 and pNN50, and the increase in sympathetic modulation in the TPG was ratified by the lower RMSSD, NN50 and PNN50 values (12). SDNN and total power parameters presented a decreased tendency in the TPG after training, opposed to the increase reached in the RPG, that evidences an increase in parasympathetic control of the sympatheticvagal balance (28).

The current data did not corroborate the assumption that the increase in autonomic modulation has been associated with increases in athletic performance (5), because the TPG decreased values of total power and slightly increased the performance in the 50-m test, and by contrary the RPG increased values of total power and decreased performance in the 50-m test. These apparent conflicting findings might be explained by sport-specific adaptations and/or training protocol differences. In addition, the decrease on total power achieved by the TPG was also reported after a marathon and after moderate exercise (9). Conversely, the RPG presented an increase in this parameter, showing a different autonomic response to acute exercise and high volumes of training. The higher autonomic adaptation achieved by the RPG was consistent with the results obtained by Sant'Ana et al. (20) demonstrating greater autonomic adaptation, after a 10 -week training period with rats, performing 30 -min swimming session, than 60-min swimming sessions. This fact confirms the efficiency of short training opposed to high volume training.

The resting HR measured in the RPG and TPG denoted a bradycardia, typical of highly trained athletes (3). In the current study, the resting HR was not modified in any of the groups after the 10 weeks of training, in contrast to the results obtained with athletes after 3 weeks of hard training (16) and also after performing an overtraining program during 20 days with athletes (3). These different results obtained might be explained by the high workload and stress imposed by these two programs compared to the current study and because they were conducted in running, rather than in swimming. However, as rest HR increase differences were small, and as HRV seems to give higher contrast to them, it seems that the measurement of HRV indices, applied to training protocols, could better evidence the organic status of the athletes than resting HR (16). In the present study, both swimmers' groups achieved a resting bradycardia before and after performing the training program, this fact would be in contrast 
to the increase in LF of the TPG that showed an increase in sympathetic modulation. The continued sympathetic activation after intense exercise might explain the coexistence of the bradycardia with the increase in sympathetic activity (5).

RPG achieved a higher autonomic adaptation with lower training volume. This finding was consistent with the results of Kiviniemi et al. (12), who found that training based on obtaining a greater autonomic response allowed better results with lower training volumes. Reverse periodization model, based on low volume and high intensity training leads to greater autonomic adaptation of swimmers compared to a traditional periodization model, based on a high volume of training. These facts and the maintenance of the 50-m swimming test suggest that autonomic adaptation did not play a decisive role in the 50-m maximal swimming test performance. With a larger sample, the tendency showed in the TPG might become significant, therefore it is supposed that anaerobic performance might improve with a traditional periodization model, despite the lower autonomic adaptations. Traditionally swimming training was focused on long endurance training, independently of the distance probe of the swimmers. In the present research we tried to analyze the effect of a training periodization that avoids high volumes of aerobic training, focusing on performing high intensity interval training (HIIT). Having analyzed the result we found that HIIT could be a good training option to improve $\mathrm{VO} 2 \mathrm{max}$ or aerobic proficiency markers as previous literature demonstrates $(4,6,17,18)$, but it is not efficient in improving anaerobic performance of swimmers. To improve anaerobic performance higher volume of alactic and lactic training should be conducted. For future works it could be hypothesized that the stress induced by traditional periodization training might be beneficial for swimmers in relatively short periods, such as the 10 weeks of training used in the current research. But a new question arises: does the traditional periodization model conducted during longer periods lead to an increased performance of swimmers or, otherwise, does it cause a decrease in the swimmers' performance due to the lower autonomic adaptation evaluated during a relatively short period of 10 weeks.

\section{Limitation of the study}

The main limitations were that the two training groups did not perform a second training period changing the training model, because we did not have the swimmers at our disposal for a longer time period, and the fact that the two groups were not submitted to equal workloads. In this study, and for the sake of greater specificity of the results, we attempted to reproduce the actual training process taking place in real swimming clubs, approaching models being conducted by coaches.

\section{Practical applications}

The autonomic response depended on the periodization training model performed. High volume traditional periodization showed less autonomic adaption than low volume and high intensity reverse periodization that showed a higher autonomic adaption. Facing these results, the use of traditional periodization training models, could be recommended for the first macrocycles of the season, as it led to a less autonomous adaption (although it presented a tendency to greater increases in performance than reverse periodization model). After performing this periodization model, a reverse periodization macrocycle should be conducted to directly prepare competitions. In this macrocycle the swimmers should perform training of higher intensity to achieve a higher performance, since subjects who performed this program in the present research presented an adaptive autonomous response. This fact has led us to 
think that it is possible to further increase the intensity of the training program without compromising the adaptive autonomic response of the swimmers. This information could help coaches to develop better training programs and correctly organize the different training macrocycles during the season to obtain a better autonomic response of their athletes prior and during competitions.

\section{Conclusion}

Autonomic response of swimmers was different depending on the periodization model performed. The reverse periodization model allowed a higher autonomic adaptation than the traditional model. It appears that autonomic adaptations might be not a determinant for short duration swimming performance.

\section{REFERENCES}

1. Clemente V, Gonzalez J: Four weeks of training with different aerobic workload distributions - Effect on aerobic performance. Eur. J. Sport Sci. DOI:10.1080/17461391.2011.635708 (2011)

2. Costill DL, Thomas R, Robergs RA, Pascoe D, Lambert C, Barr S, Fink WJ: Adaptations to swimming training: influence of training volume. Med. Sci. Sports Exerc. 23, 371-377 (1991)

3. Dressendorfer RH, Wade CE, Scaff JH: Increased morning heart rate in runners: a valid sign of overtraining?. Physician Sportmed. 13, 77-86 (1985)

4. Ebben W, Kindler A, Chirdon K, Jenkins N, Polichnowski A, Ng AV: The effect of high-load vs. high-repetition training on endurance performance. J. Strength Cond. Res. 18, 513-517 (2004)

5. Furlan R, Piazza S, Dell'Orto S, Gentile E, Cerutti S, Pagani M, Malliani A: Early and late effects of exercise and athletic training on neural mechanisms controlling heart rate. Cardiovas. Res. 27(3), $482-488$ (1993)

6. Gibala M, Little JP, Van Essen M, Wilkin GP, Burgomaster KA, Safdar A, Raha S, Tarnopolsky M: Short-term sprint interval versus traditional endurance training: similar initial adaptations in human skeletal muscle and exercise performance. J. Physiol. 575(3), 901-911 (2006)

7. Hamlin MJ, Manimmanakorn A, Sandercock GR, Ross JJ, Creasy RH, Hellemans J: Heart rate variability in responders and non-responders to live-moderate, train-low altitude training. World Acad. Sci. Eng. Tech. 77, 936-940 (2011)

8. Hopkins WG, Hawley JA, Burke LM: Design and analysis of research on sport performance enhancement. Med. Sci. Sports Exerc. 31, 472-485 (1999)

9. Hynynen E, Vesterinen V, Rusko H, Nummela A: Effects of moderate and heavy endurance exercise on nocturnal HRV. Int. J. Sports Med. 31, 428-432 (2010)

10. Iellamo F, Pigozzi F, Spataro A, Lucini D, Pagani M: T-wave and heart rate variability changes to assess training in world-class athletes. Med. Sci. Sports Exerc. 36(8), 1342-1346 (2004)

11. Issurin V: New horizons for the methodology and physiology of training periodization: Rev. Sports Med. 40(3), 189-206 (2010)

12. Kiviniemi AM, Hautala AJ, Kinnunen H, Tulppo MP: Endurance training guided individually by daily heart rate variablity measurements. Eur. J. Appl. Physiol. 101, 743-751 (2007)

13. Melanson EL, Freedson PS: The effect of endurance training on resting heart rate variability in sedentary adult males. Eur. J. Appl. Physiol. 85, 442-449 (2001)

14. Mujika I, Padilla S: Scientific bases for precompetition tapering strategies. Med. Sci. Sports Exerc. 35, 11821187 (2003)

15. Padilla S, Mujika I, Orbañanos J, Santiesteban J, Angulo F, Goiriena JJ: Exercise intensity and load during massstart stage races in professional road cycling. Med. Sci. Sports Exerc. 33, 796-802 (2001)

16. Pichot V, Roche F, Gaspoz JM, Enjolras F, Antoniadis A, Minini P, Costes F, Busso T, Lacour R, Barthelemy JC: Relation between heart rate variability and training load in middle-distance runners. Med. Sci. Sports Exerc. 32(10), 1729-1736 (2000).

17. Prestes J, De Lima C, Frollini AB, Donatto FF, Conte M: Comparison of linear and reverse linear periodization effects on maximal strength and body composition. J. Strength Cond. Res. 23(1), 266-274 (2009) 
18. Rhea MR, Ball S, Phillips WT, Burkett LN: A comparison of linear and daily undulating periodized programs with equated volume and intensity for local muscular endurance. J. Strength Cond. Res. 17(1), 82-87 ( 2003)

19. Sandercock GR, Bromley PD, Brodie DA: Effects of exercise on heart rate variability: inferences from metaanalysis. Med. Sci. Sports Exerc. 37, 433-439 (2005)

20. Sant'Ana J, Pereira M, Dias V, Dambrós C, Costa-Neto C, Souza H: Effect of the duration of daily aerobic physical training on cardiac autonomic adaptations. Autonomic Neuroscience Basic Clin. 159, 32-37 (2011)

21. Santhiago V, Da silva A, Papoti M, Gobatto C: Responses of hematological parameters and aerobic performance of elite men and women swimmers during a 14-week training program. J. Strength Cond. Res. 23(4), 1097-1105 (2009)

22. Seiler S: What is best practice for training intensity and duration distribution in endurance athletes? Int. J. Sports Physiol. Perform. 5, 276-291 (2010)

23. Task Force of the European Society of Cardiology and the North American Society of Pacing and Electrophysiology: "Heart rate variability; standards of measurement, physiological interpretation and clinical use," Circulation 93, 1043-1065 (1996)

24. Tulppo MP, Hautala AJ, Mäkikallio TH, Laukkanen RT, Nissilä S, Hughson RL, Huikuri HV: Effects of aerobic training on heart rate dynamics in sedentary subjects. J. Appl. Physiol. 95, 364-372 (2003)

25. Uusitalo A, Uusitalo J, Rusko H: Endurance training, overtraining and baroreflex sensitivity in female athletes. Clin. Physiol. 18, 510-520 (1998)

26. Verkhosansky Y: The end of "periodization" of training in top-class sport. New Stud. Athletics 14(2), 47-55 (1999)

27. Waldeck M, Lambert M: Heart rate during sleep: implications for monitoring training status. J. Sports Sci. Med. 2, 133-138 (2003)

28. Yamamoto K, Miyachi M, Saitoh T, Yoshioka A, Onodera S: Effects of endurance training on resting and postexercise cardiac autonomic control. Med. Sci. Sports Exer. 33, 1496-1502 (2001) 